\title{
Searching for a supersolid in cold-atom optical lattices
}

\section{Citation}

Scarola, V. W., E. Demler, and S. Das Sarma. 2006. "Searching for a Supersolid in Cold-Atom Optical Lattices." Physical Review A 73 (5) (May 17). doi:10.1103/physreva.73.051601.

\section{Published Version}

doi:10.1103/PhysRevA.73.051601

\section{Permanent link}

http://nrs.harvard.edu/urn-3:HUL.InstRepos:27896443

\section{Terms of Use}

This article was downloaded from Harvard University's DASH repository, and is made available under the terms and conditions applicable to Other Posted Material, as set forth at http:// nrs.harvard.edu/urn-3:HUL.InstRepos:dash.current.terms-of-use\#LAA

\section{Share Your Story}

The Harvard community has made this article openly available.

Please share how this access benefits you. Submit a story.

\section{Accessibility}




\title{
Searching for a supersolid in cold-atom optical lattices
}

\author{
V. W. Scarola, ${ }^{1}$ E. Demler, ${ }^{2}$ and S. Das Sarma ${ }^{1}$ \\ ${ }^{1}$ Condensed Matter Theory Center, Department of Physics, University of Maryland, College Park, Maryland 20742, USA \\ ${ }^{2}$ Physics Department, Harvard University, Cambridge, Massachusetts 02138, USA
}

(Received 13 February 2006; published 17 May 2006)

\begin{abstract}
We suggest a technique for the observation of a predicted supersolid phase in extended Bose-Hubbard models which are potentially realizable in cold-atom optical lattice systems. In particular, we discuss important subtleties arising from the existence of the trapping potential which lead to an externally imposed (as opposed to spontaneous) breaking of translational invariance. We show, by carefully including the trapping potential in our theoretical formalism, that noise correlations could prove instrumental in identifying the supersolid and density wave phases. We also find that the noise correlation peak width scales inversely with the relative size of trapped Mott domains.
\end{abstract}

DOI: 10.1103/PhysRevA.73.051601

Cold-atom optical lattice systems are fast becoming ideal model systems for studying quantum phases and quantum phase transitions in various strong correlation condensedmatter Hamiltonians [1]. Experimental indicators for novel quantum collective phases such as the superfluid and the Mott insulator [2-4] already exist in cold-atom systems, and there are concrete predictions under feasible and realistic experimental conditions for the existence of several other interesting collective phases including various quantum spin phases [5,6], fractional quantum Hall phases [7], and a strongly correlated supersolid cold-atom phase [8].

A key issue in the context of correlated cold-atom quantum phases is their actual experimental observability (in contrast to their possible physical existence). The experimental techniques (based mostly on interferometry) for studying cold-atom optical lattices differ vastly from the standard spectroscopic, thermodynamic, and transport measurements prevalent in quantum condensed-matter systems. Therefore, it is not always manifestly obvious how to experimentally observe and compellingly establish the various strongly correlated collective phases in cold-atom optical lattice systems even if their existence is convincingly theoretically predicted. The situation is further complicated by the existence of the slowly varying externally imposed confining trap potential which is usually present in cold-atom systems. In fact, it is known that the confining trap potential introduces special features which, if not accounted for carefully in the theory, could lead to incorrect conclusions about the quantum phase diagram of the system.

We consider the experimental observability of the predicted cold-atom supersolid phase using the recently proposed [9] and demonstrated $[4,10,11]$ noise correlation technique which is particularly suited to probing many-body states of cold atoms by directly investigating second-order correlations in the absorption images of expanding atomic gas clouds. Conceptually, the noise correlation technique is akin to solid-state neutron, electron, or x-ray spectroscopy of strongly correlated condensed-matter systems where a suitable correlation function is spectroscopically observed providing a direct measure of strong correlation effects. We show that the noise correlation function proves vital in distinguishing between spontaneous and externally broken translational symmetry in trapped cold-atom optical lattice systems.
PACS number(s): 03.75.Lm, 03.75.Nt, 32.80.Pj, 67.90.+z

We study the observable properties of two-dimensional cold-atom optical lattices in the presence of a parabolic trap modeled by the single-band, extended Bose-Hubbard Hamiltonian,

$$
\begin{aligned}
H= & -t \sum_{\langle i, j\rangle}\left(b_{i}^{\dagger} b_{j}+\text { h.c. }\right)+\frac{U}{2} \sum_{i} n_{i}\left(n_{i}-1\right)+V \sum_{\langle i, j\rangle} n_{i} n_{j} \\
& -\sum_{i} \mu_{i} n_{i},
\end{aligned}
$$

where $b_{i}^{\dagger}$ creates a boson at the site $i$ and $n_{i}=b_{i}^{\dagger} b_{i}$. The first term indicates the hopping, $t$, between nearest neighbors, denoted by angular brackets. The second term represents the on-site interaction energy cost, $U$. The third term contains the interaction energy cost, $V$, between nearest neighbors arising from the spatially extended range of interaction possibly generated from a long-range dipolar interaction $[12,13]$, a short-range interaction between bosons in higher bands of the lattice [8], or a combination thereof. A parabolic trapping potential of strength $\kappa$ externally breaks translational invariance by modifying the chemical potential: $\mu_{i}=\mu_{0}-\kappa\left|\boldsymbol{R}_{i}\right|^{2}$, where $\boldsymbol{R}_{i}=\left(i_{x}, i_{y}\right)$ for a two-dimensional square lattice. We work in units of the intersite spacing, $a$, equal to half the wavelength of the lasers defining the optical lattice.

To study the experimentally observable ground-state properties of Eq. (1) we first consider the Gutzwiller, meanfield phase diagram in the absence of a trapping potential, $\kappa=0$ [14]. We obtain the phase diagram using a variational state $[2,15,16]$ in the Fock number basis, $\left|N_{i}\right\rangle:\left|\psi_{i}\right\rangle$ $=\sum_{N_{i}=0}^{N_{m}-1} f_{N_{i}}^{i}\left|N_{i}\right\rangle$, where we fix the $S N_{m}$ variational parameters $f_{N_{i}}^{i}$ on $S$ sublattices. We find that $N_{m}=6$ ensures convergence in the calculations shown here. Evaluating $\min \left[\left\langle\psi_{i \in S}|H| \psi_{i \in S}\right\rangle\right]$ yields the phase diagram shown in Fig. 1. In the top panel we choose $V=0$ giving only two phases: the Mott phase and the superfluid phase. The incompressible Mott phase occurs at integer filling, $\nu$, with order parameter $\left\langle n_{i}\right\rangle \in$ integer while the superfluid can have variable filling with order parameter $\left\langle b_{i}\right\rangle \neq 0$. The bottom panel shows the mean-field phase diagram with $V=U / z$, where $z=4$ is the number of nearest neighbors. Two new phases appear: the incompressible density wave phase at $\nu=1 / 2$ and the supersolid phase. The density wave phase on a square lattice has 


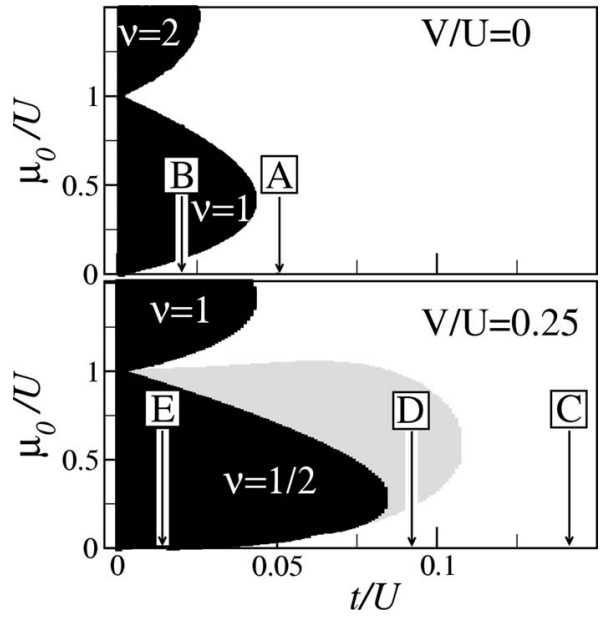

FIG. 1. Mean-field phase diagram of the Bose-Hubbard (extended Bose-Hubbard) model in the top (bottom) panel showing chemical potential vs hopping in an infinite system. The Mott and density wave phases are indicated by black at integer and half filling, $\nu$, respectively. The gray region indicates supersolid and the remaining white region superfluid. The points A through $\mathrm{E}$ label the following values of $\left(t / U, \mu_{0} / U\right)$ : $(0.05,0.6),(0.02,0.6),(0.14,0.8)$, $(0.09,0.8)$, and $(0.015,0.8)$. The arrows show the chemical-potential shifts due to an external trapping potential.

checkerboard oscillations in density with order parameter $(-1)^{\left(i_{x}+i_{y}\right)}\left(\left\langle n_{i}\right\rangle-\nu\right)$. The supersolid consists of coexisting density wave and superfluid order. Monte Carlo studies suggest that the delicate supersolid phase successfully competes with phase separation [17] for $V \gtrsim U / z$. We seek distinct experimental indicators of density wave and supersolid order as captured by a zero-temperature, modified Gutzwiller meanfield theory in a model which includes the trapping potential. The technique employed here can be extended to include finite temperature effects. In this work we focus on zerotemperature properties as a starting point.

Inclusion of the trapping potential mixes phases within the trap and may obscure experimental signatures of the phases discussed above. The center of the trap corresponds to a point in the phase diagrams of Fig. 1. The arrows indicate positions moving towards the edge of the trap which see an effectively lower chemical potential thereby mixing phases within the trap. We incorporate mixing by modifying the Gutzwiller ansatz to bring out an important realistic feature: smooth modulation of the density throughout the trap. We allow the variational parameters $f_{N_{i}}^{i}$ to vary from site to site while minimizing the energy of the total Hamiltonian: $\min [\langle\Psi|H| \Psi\rangle]$, where $|\Psi\rangle=\Pi_{i}\left|\psi_{i}\right\rangle$ generalizes $\left|\psi_{i}\right\rangle$. This leaves a minimization problem over $N_{s} N_{m}$ variables, where $N_{s}$ is the total number of sites. As an initial configuration we choose the output from our initial mean-field minimization performed independently at each site. First-order correlation functions calculated with this two-step procedure show [18] close agreement with Monte Carlo studies [19] for $V=0$ and away from the phase transition. This suggests that we retain quantitative accuracy for $V \neq 0$ although the techniques employed here remain untested against small system Monte Carlo results for $V \neq 0$. In what follows we consider system sizes comparable to experiment $N_{s}=101 \times 101$ with $\kappa / U=7$ $\times 10^{-4}$ yielding a total number of particles $N \sim 10^{3}$ for $\mu_{0} / U=0.6-0.8$ and $N=\Sigma_{i}\left\langle\Psi\left|n_{i}\right| \Psi\right\rangle$.

Time-of-flight absorption imaging of atoms released from trapped optical lattices provides a direct probe of first- and second-order correlation functions of the initially trapped state. We first consider the momentum distribution function measured through an average of images of expanding atomic clouds after a time $T$. Assuming that the particles interact weakly after the trap is adiabatically turned off, the number of particles at position $\boldsymbol{r}$ in the expanding cloud is given by [19]: $\langle n(\boldsymbol{r})\rangle_{T} \approx|\tilde{w}(\boldsymbol{Q}(\boldsymbol{r}))|^{2} \rho(\boldsymbol{Q}(\boldsymbol{r}))$, where $\boldsymbol{Q}(\boldsymbol{r})=a m \boldsymbol{r} / \hbar T$ for particles of mass $m$. The angular brackets indicate averaging, which, experimentally, occurs over several shots. $\widetilde{w}$ is the Fourier transform of the Wannier function. For illustration we take particles in the lowest band of a deep lattice. The harmonic approximation then gives (using the conventions of Ref. [8]): $|\widetilde{w}(\boldsymbol{Q})|^{2} \approx|\widetilde{w}(0)|^{2} \exp \left[-|\boldsymbol{Q}|^{2} /\left(\pi^{2} \sqrt{V_{L}}\right)\right]$, for a lattice depth $V_{L}$ in units of the photon recoil energy. $\rho$ is a firstorder correlation function related to the distribution of lattice momenta $\boldsymbol{k}$ in the trapped system,

$$
\rho(\boldsymbol{k})=\sum_{i, j} e^{i\left(\boldsymbol{R}_{i}-\boldsymbol{R}_{j}\right) \cdot \boldsymbol{k}}\left\langle b_{i}^{\dagger} b_{j}\right\rangle .
$$

Peaks in $\rho$ indicate intersite phase coherence. From shot-toshot a coherent state maintains, to a good approximation, the same phase relationship between Fourier components in the averaging process. However, for incoherent states with random Fourier components, i.e., the Mott and density wave phases, the averaging process eliminates any peak in the Fourier spectrum. One can overcome this loss of information by averaging the second-order, noise correlation function.

Noise in the imaged atomic momentum distribution provides a direct measure of higher-order correlation functions. Shot-to-shot averaging in the quantity $\left\langle n(\boldsymbol{r}) n\left(\boldsymbol{r}^{\prime}\right)\right\rangle_{T}$ $-\langle n(\boldsymbol{r})\rangle_{T}\left\langle n\left(\boldsymbol{r}^{\prime}\right)\right\rangle_{T}$ yields results proportional to momentum correlations in the ground state of the trapped system [9],

$$
\mathcal{G}\left(\boldsymbol{r}, \boldsymbol{r}^{\prime}\right) \sim|\widetilde{w}(\boldsymbol{Q}(\boldsymbol{r}))|^{2}\left|\widetilde{w}\left(\boldsymbol{Q}\left(\boldsymbol{r}^{\prime}\right)\right)\right|^{2} G\left(\boldsymbol{Q}(\boldsymbol{r}), \boldsymbol{Q}\left(\boldsymbol{r}^{\prime}\right)\right),
$$

and, in contrast to the first-order correlation function, does not vanish in finite sized system measurements of incoherent states. Higher-order terms arise in the normalized, secondorder correlation function, $\bar{G}\left(\boldsymbol{k}, \boldsymbol{k}^{\prime}\right)$, defined as

$$
\begin{aligned}
\frac{G\left(\boldsymbol{k}, \boldsymbol{k}^{\prime}\right)}{\rho(\boldsymbol{k}) \rho\left(\boldsymbol{k}^{\prime}\right)}= & \frac{\sum_{i i^{\prime} j j^{\prime}} e^{i\left(\boldsymbol{R}_{i i^{\prime}} \cdot \boldsymbol{k}+\boldsymbol{R}_{j j^{\prime}} \boldsymbol{k}^{\prime}\right)}\left\langle b_{i}^{\dagger} b_{j}^{\dagger} b_{j^{\prime}} b_{i^{\prime}}\right\rangle}{\rho(\boldsymbol{k}) \rho\left(\boldsymbol{k}^{\prime}\right)}-1 \\
& +\left(\frac{m a}{\hbar T}\right) \frac{\delta\left(\boldsymbol{k}-\boldsymbol{k}^{\prime}\right)}{\left|\widetilde{w}\left(\boldsymbol{k}^{\prime}\right)\right|^{2} \rho\left(\boldsymbol{k}^{\prime}\right)},
\end{aligned}
$$

providing a direct measure of Mott and density wave order. The delta function results from normal ordering [9]. We evaluate $\rho$ and $G$ using the output of our modified Gutzwiller mean-field theory, $|\Psi\rangle$.

In a translationally invariant system (i.e., $\kappa=0) \rho$ and $G$ qualitatively distinguish the ground states of Eq. (1). Macroscopic occupation of a Bloch mode of the optical lattice at the reciprocal-lattice vectors should lead to peaks in $\rho$ dem- 


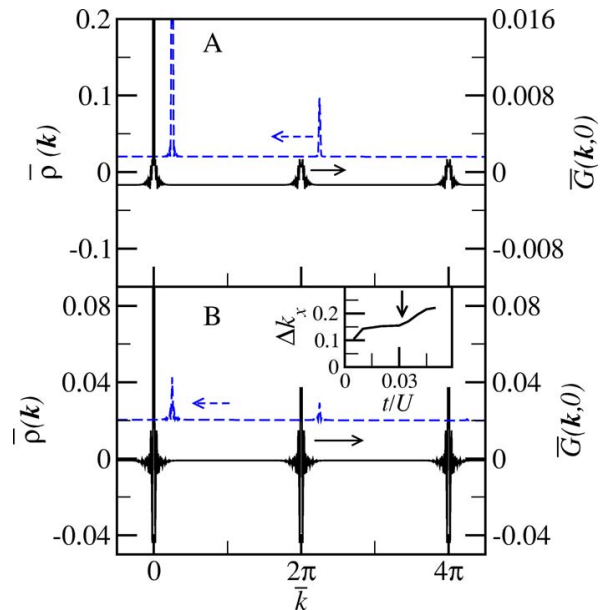

FIG. 2. (Color online) The first-order, $\bar{\rho}$, (left $y$ axis, dashed line) and second-order, $\bar{G}$, (right $y$ axis, solid line) normalized correlation functions plotted as a function of wave vector, $\bar{k}$, for the trapped Bose-Hubbard model. We set $\bar{k}=k_{x}=k_{y}$. The dashed lines are shifted up by 0.02 and right by $\pi / 4$ for clarity. The superfluid phase (top panel, point A in Fig. 1) shows strong peaks in $\bar{\rho}$ at the reciprocal-lattice vectors $\boldsymbol{K}$. In this and in the following figures we choose a deep optical lattice, $V_{L}=20$, which determines the weight of $\bar{\rho}$ at higher $\bar{k}$. The Mott phase (bottom panel, point B in Fig. 1) shows strong peaks in $\bar{G}$ at the reciprocal-lattice vectors $\boldsymbol{K}$. The inset plots the full width at half maximum of the central peak in $\bar{G}$ at $k=(2 \pi, 2 \pi)$ vs hopping for $\mu / U=0.7$. The vertical arrow indicates the position of a superfluid to Mott transition at the trap center.

onstrating a spontaneously broken $U(1)$ symmetry associated with the global phase, and therefore superfluid order. If the peak exists at wave vectors other than the reciprocal-lattice vectors we expect, in addition, spontaneously broken translational symmetry, i.e., supersolid order. (In contrast, applied potentials with spatial [20] and temporal [21] modulation of the host lattice have led to the observation of coherence peaks away from the reciprocal-lattice vectors not associated with a supersolid.) Furthermore, strong peaks in $\bar{G}$ at the reciprocal-lattice vectors of a translationally invariant optical lattice indicate Mott order while strong peaks in $\bar{G}$ at half the reciprocal-lattice vector indicate spontaneously broken translational symmetry and therefore density wave order. As we will see, both correlation functions, in a trapped system, merely quantitatively distinguish between the supersolid and other phases.

We first verify that, as expected, superfluid and Mott order in the Bose-Hubbard model remain identifiable through firstand second-order correlation functions in a trapped system. Figure 2 plots both correlation functions for $\kappa / U=7 \times 10^{-4}$ at parameters corresponding to the points A (top panel) and B (bottom panel) of Fig. 1 as a function of $\bar{k}$. The left $y$ axis plots the normalized momentum distribution function, $\bar{\rho}(\boldsymbol{k})$ $\equiv \rho(\boldsymbol{k})|\widetilde{w}(\boldsymbol{k})|^{2} /|N \widetilde{w}(0)|^{2}$, while the right $y$ axis plots $\bar{G}(\boldsymbol{k}, 0)$. The superfluid phase (top panel) shows a strong coherence peak at the reciprocal-lattice vectors $\boldsymbol{K} \equiv(2 \pi j, 2 \pi j)$, for integer $j,(\bar{\rho}(2 \pi, 2 \pi) / \bar{\rho}(0,0) \sim 0.3)$ with little signal in the

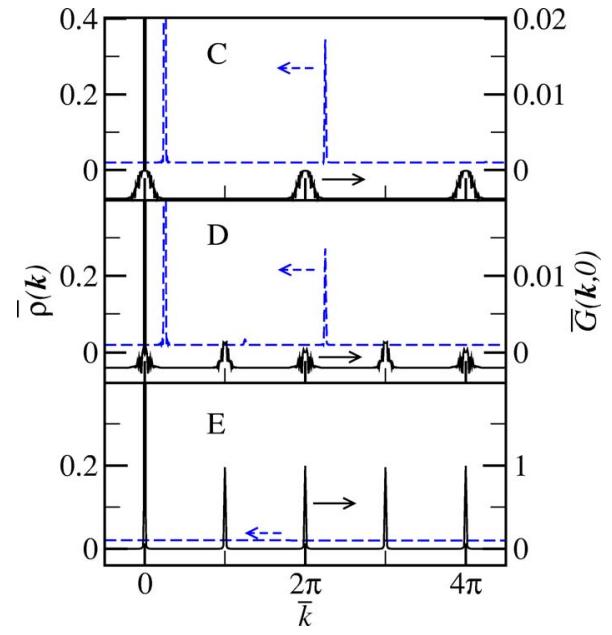

FIG. 3. (Color online) The same as Fig. 2 but for the points: C (top panel, superfluid), D (middle panel, supersolid), E (bottom panel, density wave) of Fig. 1.

noise. The exponential decay of $\widetilde{w}(\boldsymbol{k})$ strongly suppresses the peak in $\bar{\rho}$ at $(2 \pi, 2 \pi)$ as opposed to the peak at $(2 \pi, 0)$ (not shown). The Mott phase (bottom panel) is identifiable through a strong peak in $\bar{G}$, at the reciprocal-lattice vectors. Note that here there is still a small amount of superfluid at the edge of the system, identifiable through weak peaks in $\bar{\rho}$.

Individual noise correlation peaks reveal a position in the phase diagram through an additional feature, peak width. Increasing $t / U$ through the Mott phase we find the noise correlation peaks to decrease in height and split. The splitting results from our choice of normalization. Other suitably chosen normalization schemes can eliminate the splitting by excluding coherence peaks from the definition of the normalization [22]. In addition, we predict that the widths of the peaks vary inversely with the size of the Mott domains (rather than the total system size), in qualitative agreement with recent experimental results [22]. The inset to Fig. 2 shows the full width at half maximum along the $k_{x}$ axis, $\Delta k_{x}$, of the central $(2 \pi, 2 \pi)$ noise correlation peak increasing with $t / U$. An abrupt increase in peak width occurs when the center of the trap crosses from the Mott to the superfluid regime. We conclude that noise correlation peak widths yield detailed information related to the location of the system in the phase diagram.

Figure 3 shows correlation functions of the extended Bose-Hubbard model $(V / U=0.25)$ along the points $\mathrm{C}$ (top panel), D (middle panel), and E (bottom panel) of Fig. 1. The top panel shows signatures of superfluidity; a strong peak in $\bar{\rho}$, with $\bar{\rho}(2 \pi, 2 \pi) / \bar{\rho}(0,0) \sim 0.18$, and weak structure in $\bar{G}$. The bottom panel, deep in the density wave regime, shows signatures of checkerboard density wave order only; strong peaks in noise correlations at $\boldsymbol{k}=\boldsymbol{K}$ and $\boldsymbol{K} / 2$. The paucity of superfluid at the edge yields almost no structure in $\bar{\rho}$. The middle panel is plotted for parameters with supersolid at the trap center and superfluid near the edge. Here we see a coherence peak at the reciprocal-lattice vectors $\boldsymbol{K}$ and very weak structure in $\bar{G}$. There is also a very weak peak $(\sim 0.01)$ in $\bar{\rho}$ at $(\pi, \pi)$. We conclude that here weak super- 
solid order is nearly indistinguishable from superfluid order.

The supersolid shows a small signal in our mean-field estimates of the first-order correlation function. We define the strength of a supersolid phenomenologically through $\rho(\pi, \pi) / \rho(0,0)$, which is sensitive to spatial modulation of the superfluid order parameter, $\left\langle b_{i}\right\rangle$. The nearby superfluid and density wave phases surrounding the supersolid phase in parameter space show no modulation in $\left\langle b_{i}\right\rangle$. We then expect the supersolid to exhibit only weak structure in $\left\langle b_{i}\right\rangle$ and therefore $\rho(\pi, \pi)$. The modulation amplitude can be enhanced if the density wave-supersolid and superfluidsupersolid phase boundaries lie far apart in parameter space. We may therefore reach the strong supersolid regime by increasing $V / U$.

We now consider the strong supersolid regime in a trap. The main panels of Fig. 4 plot the same as Fig. 2 but for $V / U=1, \mu / U=0.8, t / U=0.2$ (top panel), and $t / U=0.1$ (bottom panel). We choose the parameters for the top panel so that the supersolid is strongest at the center of the trap with superfluid near the edge as indicated in the inset. We again see a strong, first-order coherence peak at $\boldsymbol{k}=\boldsymbol{K}$ with weak structure in $\bar{G}$. We also find $\bar{\rho}(\pi, \pi) / \bar{\rho}(0,0) \sim 0.03$.

We now compare the correlation functions of a strong supersolid with those of the trapped density wave. The bottom panel of Fig. 4 lies in the density wave regime with a significant amount of superfluid near the edge. A strong enhancement in noise correlations at $\boldsymbol{K} / 2$ signals the onset of density wave order. Superfluidity near the edge enhances $\bar{\rho}(\boldsymbol{K})$ and splits the $\bar{G}(\boldsymbol{K}, 0)$ peaks. There is also a small but surprising feature in $\bar{\rho}$ near $(\pi, \pi)$. The weak $(\pi, \pi)$ feature, $\bar{\rho}(\pi, \pi) / \bar{\rho}(0,0) \sim 0.02$, previously associated with the supersolid phase, arises here as the density wave and trap corrugate the superfluid at the edge. The modulated superfluid does not spontaneously break translational symmetry along the edge but consists of a mixture of phase boundaries mitigated by the trapping potential. We further find that the edge mode density oscillations never reach zero, as in the case of a bulk supersolid. The essential differences in going from the top panel (comprising a trapped supersolid) to the bottom

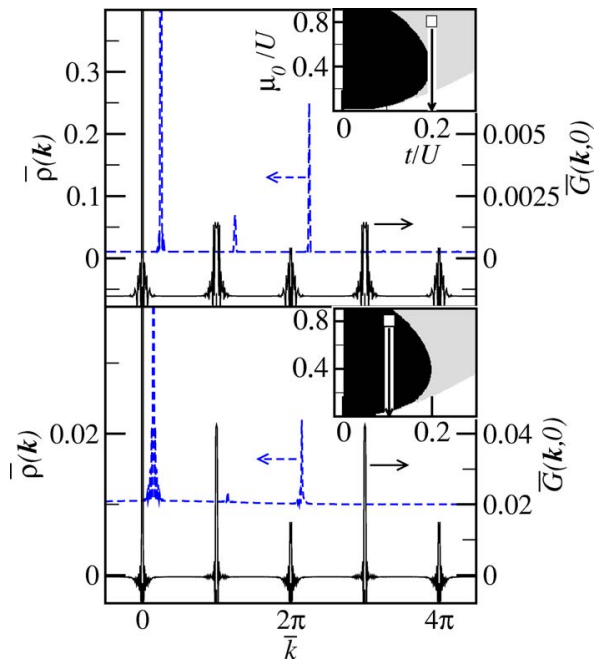

FIG. 4. (Color online) The main panels plot the same as Fig. 2 but for $V / U=1$ and $\mu / U=0.8$ with the dashed lines shifted up by 0.01 and right by $\pi / 4$ for clarity. The main top (bottom) panel is in the supersolid (density wave) regime with $t / U=0.2(t / U=0.1)$. The insets show the phase diagram (as in Fig. 1 but with $V / U=1$ ) near the $\nu=1 / 2$ density wave.

panel (a trapped density wave) are therefore merely quantitative; a large enhancement in $\bar{G}(\boldsymbol{K} / 2,0)$ combined with a sharp drop in $\bar{\rho}(\boldsymbol{K} / 2)$ and $\bar{\rho}(\boldsymbol{K})$.

Our interesting and important findings are that (1) noise correlations enable relatively precise determination of phase boundaries in trapped systems and are therefore fundamental in providing unambiguous observation of the supersolid phase, and (2) the edge of the cold-atom density wave may accommodate a superfluid component, mimicking the existence of an edge supersolid.

We thank I.B. Spielman and J.V. Porto for valuable discussions. V.S. and S.D.S. acknowledge support from AROARDA and NSA-LPS.
[1] P. Verkerk et al., Phys. Rev. Lett. 68, 3861 (1992); P. S. Jessen et al., ibid. 69, 49 (1992); A. Hemmerich and T. W. Hansch, ibid. 70, 410 (1993).

[2] D. Jaksch et al., Phys. Rev. Lett. 81, 3108 (1998).

[3] M. Greiner et al., Nature (London) 415, 39 (2002).

[4] S. Fölling et al., Nature (London) 434, 481 (2005).

[5] L. M. Duan et al., Phys. Rev. Lett. 91, 090402 (2003).

[6] A. Isacsson and S. M. Girvin, Phys. Rev. A 72, 053604 (2005).

[7] A. S. Sørensen et al., Phys. Rev. Lett. 94, 086803 (2005).

[8] V. W. Scarola and S. Das Sarma, Phys. Rev. Lett. 95, 033003 (2005).

[9] E. Altman et al., Phys. Rev. A 70, 013603 (2004).

[10] Z. Hadzibabic et al., Phys. Rev. Lett. 93, 180403 (2004).

[11] M. Greiner et al., Phys. Rev. Lett. 94, 110401 (2005).
[12] K. Goral et al., Phys. Rev. Lett. 88, 170406 (2002).

[13] A. Griesmaier et al., Phys. Rev. Lett. 94, 160401 (2005).

[14] M. P. A. Fisher et al., Phys. Rev. B 40, 546 (1989).

[15] D. S. Rokhsar and B. G. Kotliar, Phys. Rev. B 44, 10328 (1991).

[16] A. Otterlo et al., Phys. Rev. B 52, 16176 (1995).

[17] G. G. Batrouni et al., Phys. Rev. Lett. 84, 1599 (2000); P. Sengupta et al., ibid. 94, 207202 (2005).

[18] J. Zakrzewski, Phys. Rev. A 71, 043601 (2005).

[19] V. A. Kashurnikov et al., Phys. Rev. A 66, 031601(R) (2002).

[20] S. Peil et al., Phys. Rev. A 67, 051603(R) (2003).

[21] N. Gemelke et al., Phys. Rev. Lett. 95, 170404 (2005).

[22] I. B. Spielman and J. V. Porto (unpublished); (private communication). 